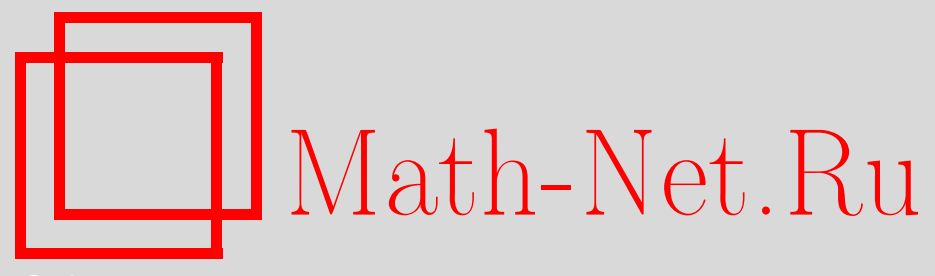

М. Королев, Обманчивая простота простых чисел, Квант, 2020, номер 3, 10-17

DOI: https://doi.org/10.4213/kvant20200302

Использование Общероссийского математического портала Math-Net.Ru подразумевает, что вы прочитали и согласны с пользовательским соглашением http://www.mathnet.ru/rus/agreement

Параметры загрузки:

IP: 54.157 .27 .8

26 апреля 2023 г., 10:11:02

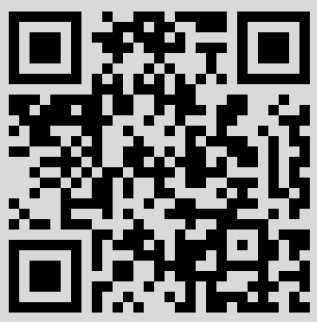




\section{Обманчивая простота простых чисел}

М.КОРОЛЕВ

$\Pi$ РОСТЫЕ ЧИСЛА.. ЧТО МЫ ЗНАЕМ о них из школьного курса? Во-первых, их определение: натуральное число $n \geq 2$ называется простым, если оно имеет только два делителя - единицу и самого себя. Во-вторых, теорему Евклида о том, что множество простых чисел бесконечно. Наконец, основную теорему арифметики, согласно которой всякое целое число $n \geq 2$ разлагается в произведение степеней простых чисел, причем единственным образом с точностью до перестановки сомножителей. Ну вот, собственно, и все.

Потому неудивительно, что название «простые» приобретает в наших глазах уничижительный оттенок. Между тем, эта «простота» обманчива, и при внимательном рассмотрении простые числа оказываются совсем даже не простыми.

К слову сказать, «простой» - результат не очень удачного перевода греческого слова «протон», что значит «первичный», «основной». ${ }^{1}$ То же название носят элементарные частицы - кирпичики нашего Мироздания. Стало быть, простые числа - это «элементарные частицы», из которых как из строительного материала построено все здание математики. Неудивительно, что знание их свойств, законов, которым они подчиняются, имеет колоссальное значение.

Задача 1. Попробуйте доказать теорему Евклида.

\section{Асимптотический закон распределения простых чисел}

В свете теоремы Евклида вопрос о том, сколько существует простых чисел, лишен

1 Кстати, в английском языке простой simple, но «простые числа» - «prime numbers» или «primes», а не «simple numbers».

DOI: https://doi.org/10.4213/kvant20200302 смысла. Однако небольшое его видоизменение сразу приводит к серьезной задаче, над решением которой ломали головы лучшие математические умы. Пусть $x \geq 0-$ произвольное (не обязательно целое) число. Обозначим через $\pi(x)$ количество простых чисел $p$, не превосходящих $x$ (рис.1). Например, $\pi(2,5)=1, \pi(5)=3, \pi(100)=25$, $\pi\left(10^{6}\right)=78498$.

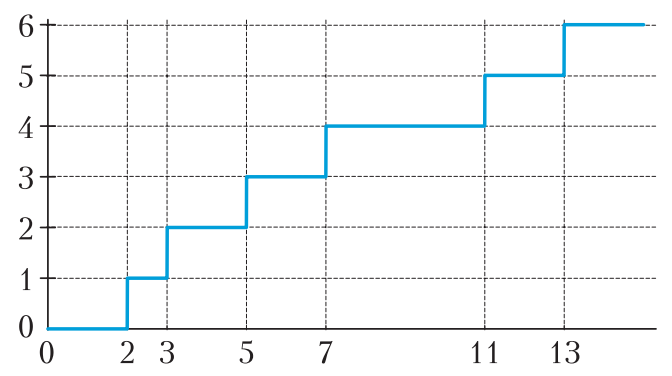

Рис. 1. График функции $\pi(x)$ на отрезке $0 \leq x \leq 15$

Как ведет себя функция $\pi(x)$ с ростом $x$ ? Из теоремы Евклида мы уже знаем, что $\pi(x)$ стремится к бесконечности при неограниченном возрастании $x$. Можно ли описать ее поведение более точно? Изучение обширных таблиц простых чисел привело в начале XIX столетия французского математика Адриена Мари Лежандра (1752-1833) к предположению о том, что

$$
\pi(x) \sim \frac{x}{\ln x} .
$$

Это соотношение нужно понимать в следующем смысле: как бы ни было мало положительное число $\varepsilon$, найдется $x_{0}$, зависящее лишь от $\varepsilon$, такое, что при всех $x \geq x_{0}$ отношение левой и правой частей (1) будет заключено между $1-\varepsilon$ и $1+\varepsilon$, или, что то же, $(1-\varepsilon) \frac{x}{\ln x}<\pi(x)<(1+\varepsilon) \frac{x}{\ln x}, x \geq x_{0}$. 
Утверждение (1) называется асимптотическим законом распределения простых чисел или, сокращенно, А.З.Р.П.Ч. Он был доказан в 1896 году независимо двумя учеными - Жаком-Саломоном Адамаром (1865-1963) и Шарлем Жаном де ла Валле-Пуссеном (1866-1962). О том, как удалось это сделать, мы расскажем чуть позже.

\section{Функции Чебышёва и Мангольдта}

Важнейшей вехой на пути к доказательству А.З.Р.П.Ч. стал мемуар «О простых числах» (1850) нашего выдающегося соотечественника Пафнутия Львовича Чебышёва (1821-1894). Он содержал строгое доказательство неравенств для функции $\pi(x)$, подобных (2), но несколько менее точных. В них вместо $1-\varepsilon$ и $1+\varepsilon$ фигурировали постоянные $a$ и $b$, причем первая была чуть меньше единицы, вторая - чуть больше: $a=0,92129 \ldots, b=$ $=1,10555 \ldots^{2}$

Еще одной заслугой Чебышёва стало введение важной функции, которая обозначается $\psi(x)$ и с тех пор носит его имя. В свою очередь, функция Чебышёва $\psi(x)$ тесно связана с еще одной арифметической функцией - на этот раз названной в честь немецкого математика Ганса Карла Фридриха фон Мангольдта (1854-1925). Поскольку обе они очень важны для дальнейшего рассказа, остановимся на них подробнее.

Функция Мангольдта $\Lambda(n)$ определяется следующим образом: если $n$ - степень простого числа, т.е. $n=p^{k}$, где $k \geq 1$, то полагают $\Lambda(n)=\ln p$; во всех остальных случаях $\Lambda(n)=0$. Вот ее значения на первом десятке натуральных чисел: $\Lambda(1)=$ $=\Lambda(6)=\Lambda(10)=0, \quad \Lambda(2)=\Lambda(4)=\Lambda(8)=$ $=\ln 2, \quad \Lambda(3)=\Lambda(9)=\ln 3, \Lambda(5)=\ln 5$ и, наконец, $\Lambda(7)=\ln 7$.

Задача 2. Докажите тождество $\sum_{d \mid n} \Lambda(d)=$ $=\ln n$ (символ $d \mid n$ означает суммирование по всем делителям $d$ числа $n$ ).

2 В 1881 году английский математик Джеймс Джозеф Сильвестр (1814-1897) установил еще более тесные границы для $\pi(x)$ при всех достаточно больших $x: a=0,95095 \ldots, b=1,04423 \ldots$
Теперь можно определить и $\psi(x)$ - это сумма значений $\Lambda(n)$ по всем натуральным числам $n$, не превосходящим $x$ :

$$
\psi(x)=\sum_{1 \leq n \leq x} \Lambda(n) .
$$

«Да чем же она хороша?»- впору спросить нам вслед за поэтом Ярославом Смеляковым. Дело в том, что А.З.Р.П.Ч. можно формулировать и в терминах $\psi(x)$, причем соответствующая формулировка выглядит существенно проще (1):

$$
\psi(x) \sim x .
$$

Задача 3. Попробуйте доказать, что утверждения (1) и (3) равносильны.

На рисунках 2 и 3 представлены графики функции Чебышёва на отрезках

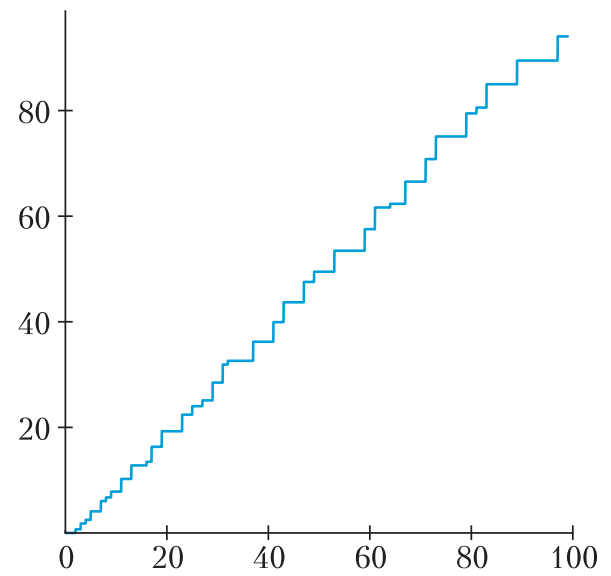

Рис. 2. График функции $\psi(x)$ на отрезке $0 \leq x \leq 100$

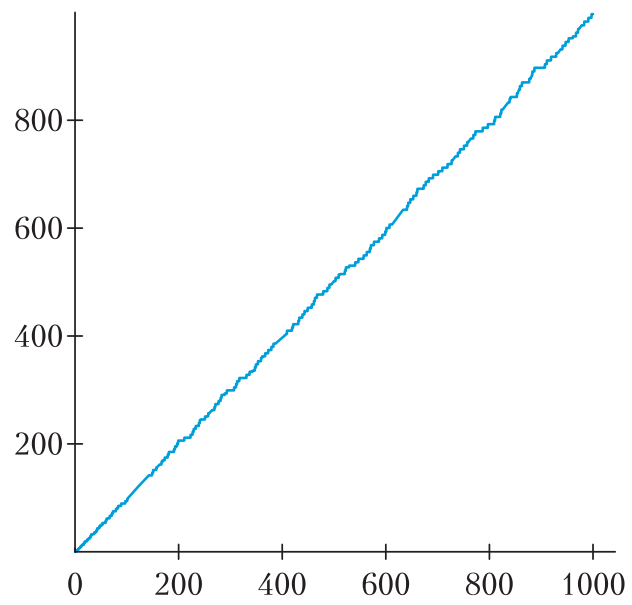

Рис. 3. График функции $\psi(x)$ на отрезке $0 \leq x \leq 1000$ 
$0 \leq x \leq X, X=100$ и $X=1000$. Если на первом из них «ступеньки», возникающие благодаря скачкам в точках вида $x=p^{k}$, видны, что называется, неворуженным глазом, то второй график практически неотличим от прямой $y=x$.

\section{Дзета-функция Римана}

Теперь самое время перейти к рассказу о том, как же был доказан А.3.Р.П.Ч. Но для этого нам нужно познакомиться с новым персонажем - дзета-функцией Римана $\zeta(s)$. Для $s>1$ значение дзета-функции определяется как сумма бесконечного числа слагаемых:

$$
\zeta(s)=\frac{1}{1^{s}}+\frac{1}{2^{s}}+\frac{1}{3^{s}}+\ldots=\sum_{n=1}^{+\infty} \frac{1}{n^{s}} .
$$

Задача 4. а) Докажите, что при любом фиксированном $s>1$ эта сумма конечна.

Замечание. Таким образом, последовательность частичных сумм

$$
S_{N}=\sum_{n=1}^{N} \frac{1}{n^{s}}, \quad N=1,2,3, \ldots
$$

монотонно возрастает и ограничена сверху и, по известной теореме курса математического анализа, имеет предел, который и объявляется значением $\zeta(s)$.

б) Докажите, что сумма

$$
\sum_{n=1}^{N} \frac{1}{n}=1+\frac{1}{2}+\frac{1}{3}+\ldots+\frac{1}{N}
$$

выбором $N$ может быть сделана большей любого наперед заданного числа (этим и объясняется ограничение $s>1$ в формуле (4)).

$$
\text { Выражения вида } \sum_{n=1}^{+\infty} a_{n} \text { называются беско- }
$$
нечными рядами. Ряды, подобные (4), встречались математикам уже в XVII столетии. Например, знаменитая «базельская задача» ${ }^{3}$, поставленная в 1644 году итальянским математиком Пьетро Менголи (1626$1686)$, состоит в нахождении точного значе-

\footnotetext{
${ }^{3}$ Название задачи объясняется тем, что она получила широкую известность среди европейских математиков лишь в конце XVII столетия благодаря Якобу Бернулли (1655-1705), профессору Базельского университета.
}

ния суммы обратных квадратов, т.е. $\zeta(2)$. Решение этой задачи, найденное в 1735 году Леонардом Эйлером (1707-1783), привело к открытию целого ряда замечательных формул, в числе которых - равенства

$$
\begin{gathered}
\zeta(2)=\frac{\pi^{2}}{6}, \zeta(4)=\frac{\pi^{4}}{90}, \\
\zeta(6)=\frac{\pi^{6}}{945}, \zeta(8)=\frac{\pi^{8}}{9450}
\end{gathered}
$$

и т.д. ${ }^{4}$ Другим замечательным открытием Эйлера стало следующее тождество, носящее теперь его имя:

$$
\begin{aligned}
\zeta(s)= & \prod_{p}\left(1-\frac{1}{p^{s}}\right)^{-1}= \\
& =\prod_{p}\left(1+\frac{1}{p^{s}}+\frac{1}{p^{2 s}}+\frac{1}{p^{3 s}}+\ldots\right), s>1 .
\end{aligned}
$$

В правой части этого тождества стоит бесконечное произведение по всем простым числам. Этот удивительный факт содержит намек на глубинную связь, которая имеется между ними и дзета-функцией.

Задача 5. Попробуйте доказать тождество Эйлера.

Возникает законный вопрос: если дзетафункцию столь плодотворно исследовал Эйлер, то почему она названа в честь Римана? Дело в том, что именно немецкий математик Бернхардт Риман (1826-1866) стал исследовать $\zeta(s)$ как функцию комплексного переменного.

\section{Немного о комплексных числах}

Здесь уместно сделать небольшое отступление и напомнить читателю о том,

${ }^{4}$ О том, как Эйлер пришел к этим удивительным равенствам, можно прочесть, например, в статье Н.Виленкина «В таинственном мире бесконечных рядов» («Квант», 1989, №10). Их строгое доказательство с помощью средств, не выходящих за рамки школьной программы, читатель сможет найти в книге А.М.Яглома и И.М.Яглома «Неэлементарные задачи в элементарном изложении» (М.: ГИТТЛ, 1954 (решение задачи №143)). 
что представляют собой комплексные числа. ${ }^{5}$ Для начала на них можно смотреть как на формальные суммы вида $z=x+i y$, где $x, y$ - вещественные числа, а $i-$ некий новый объект, который называется мнимой единицей и удовлетворяет равенству $i^{2}=-1$. Для таких сумм можно определить операции сложения, вычитания, умножения и деления так, что получится весьма стройная и красивая теория. Например, оказывается, что любой многочлен степени $n \geq 1$ имеет во множестве комплексных чисел ровно $n$ корней (с учетом кратности).

Комплексные числа удобно изображать точками на плоскости, сопоставляя числу $z=x+i y$ точку с координатами $(x ; y)$. При этом величины $x$ и $y$ называют, соответственно, вещественной и мнимой частями комплексного числа $z$, а расстояние от начала координат до такой точки, равное $\sqrt{x^{2}+y^{2}}$, называют его модулем. Горизонтальную ось, по которой откладываются вещественные части комплексных чисел, называют вещественной осью, вертикальную ось - мнимой, а саму плоскость комплексной плоскостью. Точка $(x ;-y)$, симметричная точке $(x ; y)$ относительно вещественной оси, отвечает числу $x-i y$, которое называется сопряженным к числу $z$ и обозначается $\bar{z}$.

Заметим, что привычные нам вещественные числа прекрасно чувствуют себя в новом мире: их можно представлять себе как комплексные числа с нулевой мнимой частью.

Отметим, наконец, что многие хорошо знакомые нам функции, такие как многочлен, экспонента, синус, косинус, логарифм и прочие, можно определить и для комплексного аргумента. Изучение их свойств составляет предмет теории функций комплексной переменной, имеющей массу приложений в математике и физике. Важное место в этой теории занимают

5 Более полное представление о том, что такое комплексные числа и как они применяются при решении различных задач, заинтересованный читатель может получить, например, из статьи С.Дориченко «Комплексные числа» («Квант», 2008, №5). вопросы, связанные с нахождением комплексных корней уравнений вида $f(z)=0$, где $f(z)$ - та или иная функция. Такие корни принято называть нулями $f(z)$.

\section{Дзета-функция Римана и ее нули}

Так вот, Риман указал способ, которым можно определить значение $\zeta(s)$ в любой точке $s=\sigma+i t$ комплексной плоскости, за исключением разве что $s=1$, так, чтобы получившаяся функция задавалась рядом (4) при всех вещественных $s>1$ и вместе с тем для всех $s$ сохраняла ряд важных свойств, присущих (4).

При этом оказалось, что у дзета-функции имеется бесконечно много нулей, образующих два множества. Первое составляют «тривиальные», или вещественные нули в точках вида $s=-2,-4,-6, \ldots$ Все вещественные нули имеют кратность 1 , и поведение дзета-функции в окрестности каждого из них хорошо известно.

Главную же загадку дзета-функции Римана таят нули второго множества. Все они лежат в узкой вертикальной полосе вида $0 \leq \sigma \leq 1$ и расположены симметрично относительно вещественной оси и вертикальной прямой $\sigma=0,5$ (рис.4). Иными словами, если $\rho=\beta+i \gamma-$ такой нуль, то нулями $\zeta(s)$ будут и точки

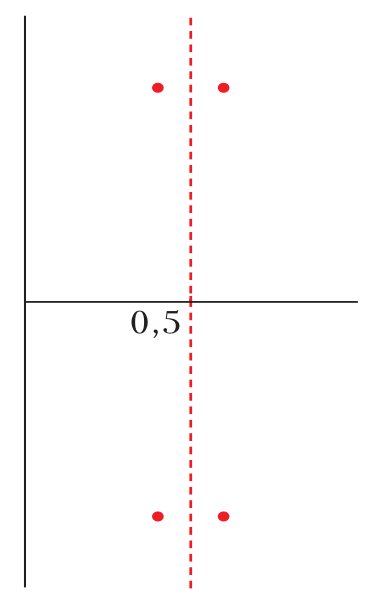

Рис. 4. Гипотетическая симметрия нулей дзетафункции Римана: если $\zeta(s)$ имеет нуль вне критической прямой, то точки, симметричные ему, также будут нулями 


$$
\begin{gathered}
\bar{\rho}=\beta-i \gamma, 1-\bar{\rho}=1-\beta+i \gamma \\
1-\rho=1-\beta-i \gamma .
\end{gathered}
$$

Вот эти-то загадочные нули, называемые «нетривиальными», или комплексными, тесно связанны с чисто арифметическими объектами - простыми числами. И первым, кто эту связь обнаружил, был Риман. Он вывел точную формулу, которая выражает функцию $\pi(x)$ через нули $\zeta(s)$.

Так как формула Римана в первоначальном ее виде достаточно сложна, мы приведем ее аналог для функции Чебышёва. Если $x>1-$ нецелое число, то

$\psi(x)=x-\sum_{\rho} \frac{x^{\rho}}{\rho}-\frac{1}{2} \ln \left(1-\frac{1}{x^{2}}\right)-\ln 2 \pi,(5)$

где в правой части стоит бесконечная сумма по всем нетривиальным нулям.

Сразу отметим, что в таком виде эту формулу почти не используют: управиться с бесконечным числом столь необычных слагаемых достаточно трудно. Поэтому (5) заменяют менее точной, но более удобной формулой, в которой участвует лишь конечное число нулей. Именно, если $x \geq 2$, а $T$ - произвольный параметр, подчиненный условиям $2 \leq T \leq x$, то

$$
\begin{aligned}
\psi(x)=x-\sum_{|\gamma| \leq T} \frac{x^{\rho}}{\rho}+r(x, T), \\
|r(x, T)| \leq c \frac{x}{T}(\ln x)^{2},
\end{aligned}
$$

где $c$ - некоторая постоянная. (Напомним, что $\gamma$ - мнимая часть $\rho$.) Таким образом,

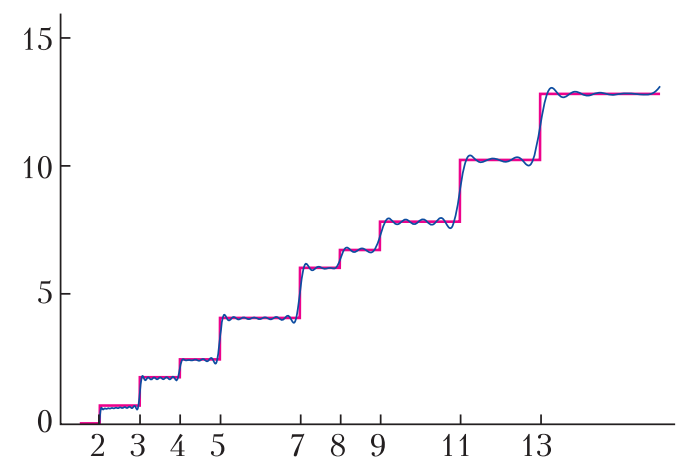

Рис. 5. Приближение функции Чебышёва, в котором участвуют первые 50 нулей дзетафункции суммирование ведется по всем нулям дзета-функции, чья мнимая часть не превосходит $T$ (рис.5).

\section{Доказательство А.З.Р.П.Ч.}

Введем еще одно обозначение: $R(x)=$ $=\psi(x)-x$. Несложно сообразить, что А.3.Р.П.Ч. будет доказан, если показать, чTO

$$
|R(x)|<\varepsilon x
$$

для сколь угодно малой постоянной $\varepsilon$ и всех $x \geq x_{0}(\varepsilon)$. Но формула (6) как раз дает явное выражение для $R(x)$. Попробуем понять, как можно воспользоваться им для доказательства (7).

Вновь полагая $\rho=\beta+i \gamma$, будем иметь

$$
\frac{x^{\rho}}{\rho}=\frac{x^{\beta} \cdot x^{i \gamma}}{\beta+i \gamma}=\frac{x^{\beta} \cdot e^{i \gamma \ln x}}{\beta+i \gamma} .
$$

Согласно формуле Эйлера,

$$
e^{i \varphi}=\cos \varphi+i \sin \varphi .
$$

Вспоминая определение модуля комплексного числа, получаем

$$
\left|e^{i \varphi}\right|=\sqrt{(\cos \varphi)^{2}+(\sin \varphi)^{2}}=1 .
$$

Следовательно,

$$
\left|\frac{x^{\rho}}{\rho}\right|=\frac{x^{\beta}}{\sqrt{\beta^{2}+\gamma^{2}}} \leq \frac{x^{\beta}}{|\gamma|}
$$

и, стало быть,

$$
|R(x)| \leq 2 \sum_{0<\gamma \leq T} \frac{x^{\beta}}{\gamma}+c \frac{x}{T}(\ln x)^{2} .
$$

Коэффициент 2 нужен для того, чтобы учесть симметрию нулей: для каждого нуля с мнимой частью $\gamma$ будет и симметричный нуль с мнимой частью $(-\gamma)$. На последнее слагаемое можно в данном случае не обращать внимания: беря $T$ достаточно большим, его можно сделать исчезающе малым по сравнению с $x$. Опасности, что в знаменателе суммируемых дробей окажется величина, близкая к нулю, нет: наименьшая из положительных ординат $\gamma$ приближенно равна $14,13 \ldots$, что было известно еще Риману. Наконец, если $\beta_{0}$ 
наибольшая из вещественных частей $\beta$ тех нулей, мнимая часть которых не превосходит $T$, то

$$
|R(x)| \ll x^{\beta_{0}} \sum_{0<\gamma \leq T} \frac{1}{\gamma} .
$$

Символ « называется знаком Виноградова. Этот знак употребляется тогда, когда математикам неохота вычислять точную константу в подобных неравенствах. Более точно, пишут $A \ll B$, если $|A| \leq c B$ для некоторой положительной постоянной c. Это удобно: позволяет сосредоточиться на главном и не отвлекаться на детали.

Кстати, о главном: сумма величин, обратных ординатам в (8), не доставляет особых проблем; можно показать (мы делать этого не будем), что она не превосходит по порядку $(\ln T)^{2}$. Поэтому главное величины $\beta$ : вся трудность - в них!

Как уже говорилось выше, все нетривиальные нули «живут» в полосе $0 \leq \beta \leq 1$. Но такая оценка для нас бесполезна: она «перевесит» главный член в выражении для $\psi(x)$, т.е. $x$. Вот если бы удалось ее улучшить...

Оказывается, чтобы доказать А.З.Р.П.Ч., достаточно «всего лишь» строгого неравенства $\beta<1$ или, что то же, достаточно доказать, что дзета-функция Римана не имеет нулей на «единичной прямой» $\sigma=1$. Именно это и было сделано Адамаром. Валле-Пуссен смог установить более точный факт: оказывается, $\zeta(s)$ не имеет нулей и в некоторой (хотя и очень узкой) окрестности единичной прямой вида

$$
\sigma \geq 1-\frac{c_{0}}{\ln |t|},|t|>14
$$

где $c_{0}$ - положительная постоянная (рис.6). Ширина этой окрестности становится исчезающее малой по мере продвижения вверх (или, наоборот, вниз) по единичной прямой. Тем не менее, этот факт позволил получить для остаточного члена $R(x)$ оценку, гораздо более точную, чем (6), а именно:

$$
R(x) \ll x e^{-c \sqrt{\ln x}},
$$

где, как обычно, $c$ - некоторая константа (ее можно выразить через $c_{0}$ ). Несложно

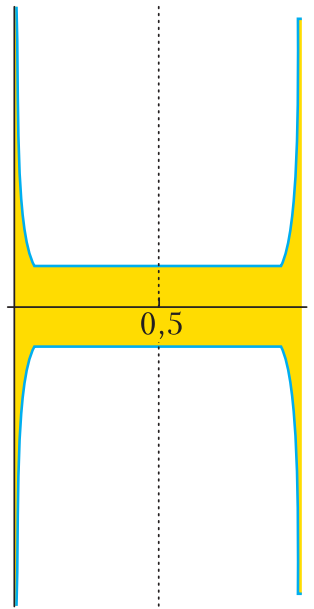

Рис. 6. Область, “свободная» от нулей

видеть (проверьте это!), что правая часть (10) хотя и растет быстрее любой функции вида $x^{1-\delta}$, но делает это куда медленнее, чем всякая функция $x(\ln x)^{-A} \quad$ (здесь $0<\delta<1, A>0-$ произвольные постоянные).

Граница (9) получена более 120 лет назад. С тех пор наши знания о нулях $\zeta(s)$ существенно пополнились, и неравенство (10) теперь можно заменить более точным:

$$
R(x) \ll x \exp \left(-\frac{(\ln x)^{0,6}}{58(\ln \ln x)^{0,2}}\right)
$$

\section{И это все?!}

«И это все?!» - спросите вы. По сути, да: за все это время показатель степени логарифма удалось сдвинуть, грубо говоря, на одну десятую, с 0,5 до 0,6 (если не обращать внимания на двойной логарифм) ${ }^{6}$. Но для этого потребовались усилия боль-

6 у математиков даже есть поговорка: все знают, что двойной логарифм стремится к бесконечности, но никто не видел, как он это делает. Хотя функция $y=\ln \ln x$ может принимать сколь угодно большие значения, в области вычислений, доступной современным компьютерам, ее рост практически незаметен. Так, например, при изменении $x$ от 10 до $10^{30}$ величина $y$ изменяется примерно с 0,83 до 4,23 . 


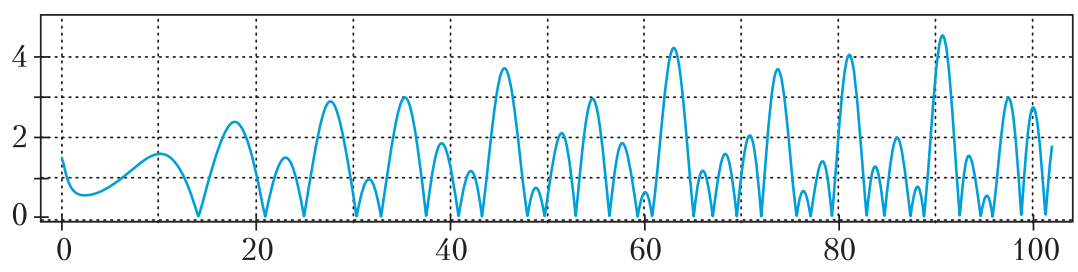

Рис. 7. Дзета-функция Римана на критической прямой - график функции $|\zeta(0,5+i t)|$ на отрезке $0 \leq t \leq 102$

шого числа ученых. Решающую роль в этой деятельности сыграл метод тригонометрических сумм, созданный выдающимся советским математиком Иваном Матвеевичем Виноградовым (1891-1983). Метод Виноградова нашел применение и в решении огромного числа других задач теории чисел.

Итак, распределение простых чисел диктуется расположением нулей дзета-функции Римана. Вычисления, начало которым положил сам Риман, показывают, что все известные к настоящему времени нули (а таких уже более 10 триллионов!) ${ }^{7}$ имеют одну и ту же вещественную часть: $\beta=0,5$ (рис.7). Риман предположил, что этим свойством обладают абсолютно все нетривиальные нули $\zeta(s)$, и это предположение, известное сейчас всему математическому миру как гипотеза Римана, до сих пор не доказано и не опровергнуто.

Гипотеза Римана вошла в число «проблем Гильберта» - кардинальных математических задач, представленных Давидом Гильбертом (1862-1943) на II Международном конгрессе математиков в Париже в 1900 году. Спустя ровно сто лет она единственная из всего этого списка оказалась в числе семи «проблем тысячелетия», отобранных Математическим институтом Клея.

Будет ли доказана гипотеза Римана в обозримом будущем или нет - непонятно. Но математикам уже сейчас интересно, что получится, если она все же верна. Посмотрим, например, что даст гипотеза Римана в задаче об оценке $R(x)$. Полагая $T=x$

\footnotetext{
${ }^{7}$ Стоит заметить, что ордината нуля с номером $n=10^{13}$ немногим меньше двух с половиной триллионов.
}

в неравенстве (8), получим

$$
R(x) \ll \sqrt{x}(\ln x)^{2} .
$$

Эта оценка по своей точности оставляет далеко позади все современные результаты. К слову, улучшить ее практически невозможно: это доказал в 1914 году английский математик Джон Идензор Литтлвуд (1885-1977).

Обратим внимание и на то, что неравенство (12) куда приятней для глаз, чем неудобоваримые экспоненты в оценках (10) и (11).

Такой же эффект наблюдается и во многих других задачах теории чисел, так или иначе связанных с нулями дзета-функции. Это, по мнению некоторых ученых, является лишним подтверждением справедливости гипотезы Римана.

Знание свойств нулей $\zeta(s)$ помогает решать и другие задачи теории простых чисел. Пусть, например, $x$ неограниченно возрастает. При каком наименьшем $h$ (как функции $x)$ промежуток $(x ; x+h]$ гарантировано будет содержать простые числа? При $h=x$ соответствующее утверждение было высказано в 1845 году французским математиком Жозефом Бертраном (1822-1900) и спустя пять лет доказано Чебышёвым. Из (10) и (11) существование простых чисел на таком «коротком» промежутке будет следовать из А.3.Р.П.Ч., если только величину $h$ взять чуть большей, чем правые части неравенств для $R(x)$. Наконец, гипотеза Римана дает утвердительный ответ на этот вопрос уже при $h \geq c \sqrt{x}(\ln x)^{2}$, если только постоянная $c$ достаточно велика. Но можно ли утверждать что-то подобное, скажем, в случае $h=x^{0,99}$, не опираясь на гипотезу Римана? 


\begin{tabular}{|l|l|l|l|}
\hline 1 & 14,134725142 & 16 & 67,079810529 \\
\hline 2 & 21,022039639 & 17 & 69,546401711 \\
\hline 3 & 25,010857580 & 18 & 72,067157674 \\
\hline 4 & 30,424876126 & 19 & 75,704690699 \\
\hline 5 & 32,935061588 & 20 & 77,144840069 \\
\hline 6 & 37,586178159 & 21 & 79,33737502 \\
\hline 7 & 40,918719012 & 22 & 82,910380854 \\
\hline 8 & 43,327073281 & 23 & 84,735492981 \\
\hline 9 & 48,005150881 & 24 & 87,425274613 \\
\hline 10 & 49,773832478 & 25 & 88,809111208 \\
\hline 11 & 52,970321478 & 26 & 92,491899271 \\
\hline 12 & 56,446247697 & 27 & 94,651344041 \\
\hline 13 & 59,347044003 & 28 & 95,870634228 \\
\hline 14 & 60,831778525 & 29 & 98,831194218 \\
\hline 15 & 65,112544048 & 30 & 101,317851006 \\
\hline
\end{tabular}

Первые 30 положительных ординат нулей дзета-функции Римана

Оказывается, можно! Более точно, можно доказать аналог А.З.Р.П.Ч. вида

$$
\pi(x+h)-\pi(x) \sim \frac{h}{\ln x}
$$

уже при $h \geq x^{a}$, где $1 \geq a>7 / 12$ - произвольная постоянная. Соотношение (13) нужно понимать так: зафиксировав любое число $a$ из этого промежутка и задавшись любой сколь угодно малой постоянной $\varepsilon>0$, можно указать величину $x_{0}$, зависящую только от $a$ и $\varepsilon$, такую, что при любом $x \geq x_{0}$ и любом $h, x^{a} \leq h \leq x$, отношение левой части (13) к правой будет заключено между $1-\varepsilon$ и $1+\varepsilon$. Иначе говоря, «короткий» промежуток $(x ; x+h]$ будет содержать громадное количество простых чисел.

Обратите внимание: 7/12 отделено от $1 / 2$ (которую дает гипотеза Римана) на сущий пустяк: $1 / 12$ ! Дело в том, что если остаточный член $R(x)$ в А.З.Р.П.Ч. может «испортить» один-единственный нуль с боль-

шой вещественной частью (точнее, пара симметричных нулей), то в задаче о простых числах на коротком промежутке это уже не так важно. Здесь главную роль играет то, насколько «плотно» расположены такие нули. Иначе говоря, для этой задачи необходимы «плотностные теоремы» - верхние оценки количества возможных нулей $\zeta(s)$, вещественная часть $\beta$ которых не превосходит заданной величины $\sigma, 0,5 \leq \sigma<1$, а мнимая часть не превосходит растущего параметра $T$. Первые утверждения такого рода были доказаны еще в 1930-е годы. К настоящему времени получено большое количество различных плотностных теорем, которые играют важную роль в теории чисел.

Доказательство гипотезы Римана привело бы к значительным продвижениям в решении многих математических проблем. В настоящее время известно большое количество «условных» утверждений, которые нетерпеливые математики доказывают в предположении («при условии»), что гипотеза Римана верна. Примером условного утверждения служит оценка (12). Однако не стоит думать, что появление доказательства этой удивительной гипотезы оставит без работы специалистов по теории чисел. На самом деле имеется много вопросов, пролить свет на которые не в силах даже она. В их числе - гипотеза о простых числах-близнецах, согласно которой существует бесконечно много простых чисел $p$, для которых число $p+2$ также будет простым; бинарная гипотеза Гольдбаха, утверждающая, что всякое четное натуральное число, большее 4, есть сумма двух нечетных простых чисел; гипотеза Лежандра о том, что между любыми двумя квадратами натуральных чисел имеется по крайней мере одно простое; вопросы бесконечности множеств простых чисел вида $n^{2}+1$ и $2^{n}-1$ и еще многое-многое другое! 